\title{
Problemas del sistema de salud en estados de México con alta incidencia de mortalidad materna
}

\author{
Mariel Rouvier, M Sc,(I) Miguel Ángel González-Block, PhD, (1) Paola Sesia, PhD,(2) Víctor Becerril-Montekio, M en Soc.(I)
}

Rouvier M, González-Block MA, Sesia P, Becerril-Montekio V.
Problemas del sistema de salud en estados de México
con alta incidencia de mortalidad materna.
Salud Publica Mex 2013;55:185-192.

\section{Resumen}

Objetivo. Identificar y priorizar problemas de los sistemas estatales de salud que limitan la eficacia de las intervenciones para prevenir la mortalidad materna. Material y métodos. Se realizó un mapeo conceptual de los problemas prioritarios percibidos por comunidades de práctica (Cop) en estados con alta incidencia de mortalidad materna. Posteriormente, las Cop revisaron la literatura médica para contrastar los problemas identificados. Resultados. Los problemas priorizados por las Cop se enfocaron en la atención de la emergencia obstétrica (AEO), específicamente en recursos financieros y desabasto de insumos (Guerrero), falta de capacitación del personal para atender la EO (Estado de México), ineficiencia de las redes obstétricas (Oaxaca) y desconocimiento y falta de adherencia a protocolos de AEO (Veracruz). La revisión de literatura médica confirmó la pertinencia de los problemas identificados mediante el mapeo conceptual. Conclusión. Las Cop pusieron en evidencia un alto grado de congruencia entre la percepción de los problemas de los sistemas estatales de salud y la evidencia científica internacional. Los problemas identificados y reformulados con base en la evidencia facilitan la identificación de soluciones apropiadas.

Palabras clave: mortalidad materna; sistemas de salud; gestión del conocimiento; México
Rouvier M, González-Block MA, Sesia P, Becerril-Montekio V. Problems of the health system in Mexican states with high incidence of maternal mortality.

Salud Publica Mex 2013;55:185-192.

\begin{abstract}
Objective. To identify and prioritize problems in states' health systems which limit the efficacy of interventions to prevent maternal mortality. Materials and methods. We made a conceptual mapping of priority problems perceived as such by communities of practice (COP) in four states with high ratios of maternal mortality in Mexico. Then, the four COP reviewed the literature and refined their formulation of previously identified problems. Results. Priority problems focused on emergency obstetric care $(\mathrm{EmOC})$, specifically: inadequate financial resources (Guerrero), substandard training among available EmOC providers (State of Mexico), inefficiencies in existing EmOC networks (Oaxaca) and inadequate knowledge of, and/or compliance to, standard EmOC protocols (Veracruz). The literature review confirmed the pertinence of problems previously identified by COP through conceptual mapping. Conclusions. The four COP showed a high level of congruency between their original perception of problems in the states' health systems and international scientific evidence. Identified problems and their reformulation based on evidence help identify solutions adaptable to local contexts.
\end{abstract}

Keywords: maternal mortality; health systems; knowledge management; Mexico

(I) Instituto Nacional de Salud Pública. Cuernavaca, Morelos, México

(2) Centro de Investigaciones y Estudios Superiores en Antropología Social, Unidad Pacífico Sur. Oaxaca, Oaxaca, México

Fecha de recibido: 7 de febrero de 2012 - Fecha de aceptado: 29 de noviembre de 2012

Autor de correspondencia: Dr. Miguel Angel González Block. Centro de Investigación en Sistemas de Salud,

Instituto Nacional de Salud Pública. Av. Universidad 655, col. Santa María Ahuacatitlán. 62100 Cuernavaca, Morelos, México.

Correo electrónico: miguel.gonzalezblock@insp.mx 
$E^{1}$ quinto objetivo del milenio propone reducir en tres cuartas partes la razón de mortalidad materna (RMM) entre 1990 y 2015. ${ }^{1}$ Para México, la meta significa disminuir la razón de 49.9 en 2011 a 22.2. ${ }^{2}$ Sin embargo, existen importantes diferencias entre los estados. En 2011 Guerrero superó una RMM de 100, mientras que en Tamaulipas no llega a 20. ${ }^{2}$ Además, en 2010, 91.1\% de las muertes maternas contó con asistencia médica y 73.1\% representó fallecimientos en unidades públicas de salud, datos que nos obligan a reflexionar acerca de las estrategias a seguir. ${ }^{3}$

El programa Arranque Parejo en la Vida (APV), implementado en México desde 2001, ha incorporado acciones para favorecer la coordinación de una red que fortalezca la organización comunitaria y mejore la referencia oportuna de mujeres embarazadas a los servicios de salud. ${ }^{4}$ El Sistema de Protección Social en Salud (SSPS) lanzó en 2008 el programa Embarazo Saludable para mejorar el acceso gratuito a la atención prenatal del embarazo, del parto y puerperio fisiológico y de cesárea y puerperio quirúrgico, sobre todo en casos de emergencias obstétricas (EO). ${ }^{5}$ En 2009, la Secretaría de Salud (SSa) federal impulsó la firma del Convenio General de Colaboración para la Atención de la Emergencia Obstétrica (AEO) entre las principales instituciones de salud. ${ }^{6}$

El Centro Nacional de Equidad de Género y Salud Reproductiva (CNEGySR), responsable de las estrategias de reducción de la RMM, ha propuesto estrategias basadas en la mejor evidencia internacional: lograr una calidad óptima en la atención médica; acceso universal y gratuito a la atención obstétrica con sistemas de comunicación disponibles y caminos transitables; y reducción de las inequidades de género y etnia, a fin de asegurar una decisión temprana en la búsqueda de atención de las mujeres y familiares, o para la prevención de los embarazos no deseados. ${ }^{7}$ El Comité Nacional por una Maternidad Segura, así como los comités estatales respectivos en Chiapas, Guerrero, Oaxaca y Veracruz, convoca anualmente a diferentes actores para generar acuerdos e implementar estrategias que contribuyan a lograr una maternidad segura y al abatimiento de la mortalidad materna. ${ }^{8}$

No obstante, la RMM sigue siendo alta y muestra un descenso lento y con altibajos. En 2011, el mayor número de muertes maternas ocurrió en el Estado de México: 145, equivalentes a 15.2\% del total; seguido por Veracruz con 80 muertes, es decir $8.4 \% .^{2}$

Este trabajo presenta resultados de un proyecto de investigación para identificar recomendaciones que permitan fortalecer la capacidad de las autoridades estatales para responder a las necesidades más relevantes en salud materna. Para ello se puso a prueba un modelo de capacitación y desarrollo de actores estatales para la identificación y fundamentación científica de problemas y recomendaciones. Se muestran los resultados a los que llegó el conjunto de actores y que contribuyeron a mejorar su comprensión de la problemática de la mortalidad materna. El proyecto se enfocó en estados de alta RMM o alta incidencia numérica. Los problemas priorizados por las Cop en cada estado fueron elegidos a partir de un ejercicio de traducción del conocimiento cuya metodología y resultados preliminares para el mapeo conceptual fueron publicados previamente. ${ }^{9-13}$

\section{Material y métodos}

El proyecto se llevó a cabo entre enero de 2010 y mayo de 2011 en Guerrero, Estado de México, Oaxaca y Veracruz. El protocolo para la investigación fue evaluado por los comités de investigación, de ética y de bioseguridad del Instituto Nacional de Salud Pública de México (INSP). En cada estado se conformó una comunidad de práctica (Cop), definida como la colaboración continua de un grupo de personas que comparten intereses, agenda y objetivos. ${ }^{14}$ Las Cop estuvieron compuestas por tomadores de decisiones, académicos, funcionarios de programas y personal tanto de los programas de salud sexual y reproductiva, como de áreas transversales (calidad, planeación y finanzas). La mayoría de los participantes laboraban para los servicios estatales de salud. El interés común fue identificar y priorizar problemas del sistema de salud que limitan la implementación de estrategias de probada efectividad para reducir la RMM. Las Cop posteriormente definieron soluciones, esfuerzo que no es informado en este artículo.

Inicialmente, la Cop de cada estado identificó y priorizó una serie de problemas del sistema de salud utilizando el mapeo conceptual e interpretando los resultados en un documento preliminar. ${ }^{9-11}$ Seleccionaron dichos problemas en función de su conocimiento tácito y posteriormente revisaron la evidencia científica internacional y la literatura gris con el fin de sustentar y reformular la selección. Las Cop fueron capacitadas por los investigadores para realizar búsquedas en bases de datos de revistas científicas con acceso en línea al texto completo, disponibles en Ebsco Host, que tiene una base de más de 6000 publicaciones científicas. Los artículos identificados fueron puestos a disposición de cada Cop para discutirlos en una plataforma virtual de gestión del conocimiento (PGC).

Los participantes seleccionaron cada artículo con base en una lectura crítica de los resúmenes y al menos dos personas decidieron sobre su pertinencia. Posteriormente, leyeron y analizaron los artículos seleccionados, 
con base en su capacitación en la interpretación de la literatura médica para la formulación de los problemas del sistema de salud utilizando diferentes marcos analíticos como antecedentes históricos, diagramas de causalidad y pertinencia a los contextos estatales. Cada Cop contó con el apoyo técnico de especialistas del INSP para garantizar el manejo efectivo de las herramientas de análisis. En conjunto se identificaron 170 artículos y documentos pertinentes para los problemas priorizados.

Posteriormente se evaluaron las coincidencias y diferencias entre la formulación de problemas con base en la percepción de las Cop y la reformulación basada en evidencia. Para ello se exploraron sistemáticamente ambas formulaciones haciendo hincapié en las especificaciones, aclaraciones, aportaciones y recomendaciones en cada caso. Utilizando como código los problemas seleccionados con base en el conocimiento tácito, se implementó la técnica del método comparativo para revisar minuciosamente las formulaciones finales. ${ }^{15}$

\section{Resultados}

La reformulación de los problemas con base en la evidencia científica generalmente corroboró la selección realizada con base en la percepción inicial de los actores. La revisión de la literatura permitió además, complementar y precisar los problemas (cuadro I). ${ }^{16-32}$

\section{Guerrero}

La revisión de literatura confirma el problema escogido por la Cop de la insuficiencia de recursos financieros como obstáculo para la AEO. Se requieren recursos financieros adicionales a aquéllos con que comúnmente cuentan los sistemas de salud en países en desarrollo y en zonas de alta pobreza para disponer de infraestructura adecuada y de medicamentos e insumos para la atención materna. ${ }^{33-35}$ Además, la inversión en programas para mejorar la salud materna tiene una alta razón de costo-efectividad en términos de años de vida saludable..$^{36}$ Diversos estudios abocados a estudiar la asignación de recursos y la implementación del SSPS en Chiapas, Guerrero y Oaxaca resaltan que uno de los principales retos que enfrenta este programa para mejorar la atención a la salud materna es la inequidad en la distribución del gasto y las fallas e inconsistencias en el avance en infraestructura y suministros. ${ }^{16}$

La Cop también identificó problemas de planeación en el ejercicio presupuestal y en el esquema de adquisiciones de bienes e insumos. La evidencia científica ratificó este problema, precisando que sus determinantes incluyen la falta de planeación en función de la deman- da, la inoportunidad en la adquisición y distribución, el alto porcentaje de claves desiertas en procesos licitatorios, la asignación inadecuada de inventarios, la baja frecuencia de entrega hacia puntos de consumo, la falta de transparencia en todos los procesos y la poca o nula comunicación e intercambio de información entre las distintas áreas involucradas en la cadena de suministro. ${ }^{17}$ De acuerdo con la literatura científica además destaca la necesidad de recursos humanos para la atención de partos los 365 días, las 24 horas, para la AEO oportuna. ${ }^{7,25} \mathrm{La}$ falta de cobertura es particularmente grave en áreas rurales de Guerrero, donde uno de cada tres partos no es atendido por personal médico y existe una escasa disponibilidad de recursos para cubrir guardias, lo que se asocia con el incremento de las defunciones en fines de semana y periodos vacacionales. ${ }^{18}$

\section{Estado de México}

La revisión de la literatura científica confirmó la relevancia del problema elegido por la Cop mexiquense en relación con asegurar la capacitación adecuada del personal de salud para abatir la RMM, particularmente en la AEO. ${ }^{19,37-40}$ También corroboró el problema seleccionado de la incorporación de profesionales de la salud con carencias técnicas al sistema de salud, ${ }^{19-21}$ lo que dificulta muchas veces el acceso de la población a servicios de salud resolutivos y de calidad, ${ }^{19}$ y especificó la necesidad de que mejoren los conocimientos básicos sobre prevención de infecciones y uso adecuado de protocolos, guías y partógrafos ${ }^{20}$ así como de normas, lineamientos técnicos y manuales de procedimiento durante la EO. 21,22

La revisión bibliográfica muestra además que los recursos humanos calificados para proporcionar atención obstétrica y tratamientos de urgencia generalmente se encuentran distribuidos de manera heterogénea. Las zonas marginadas dependen principalmente de personal que no está bien preparado para la atención de problemas obstétricos. ${ }^{16,18}$ Esto se relaciona también con la carencia de sistemas de incentivos para que estos profesionales tengan capacitación,; ${ }^{23}$ el adecuado entrenamiento de pregrado de médicos y enfermeras es también esencial para la atención de las complicaciones. ${ }^{41}$ En relación con el problema de falta de calidad en la atención, destaca la necesidad de mejorar las habilidades técnicas del personal en la AEO. ${ }^{41}$

La Cop no encontró sustento en la literatura para corroborar la notificación tardía de la preclampsia y eclampsia en el primer nivel; esto constituye la única excepción en la cual no hay clara complementación entre conocimiento tácito y publicaciones científicas. 


\section{Cuadro I \\ Comparación ENTRE LOS PROBLEMAS SELECCIONADOS DE ACUERDO AL CONOCIMIENTO TÁCITO Y A LAS REFERENCIAS halladas en la evidencia científica, por estado y problema. Proyecto de inVestigación INDESES II. Guerrero, Estado de México, Oaxaca y Veracruz, junio - diciembre de 2010}

Problema seleccionado a partir del conocimiento tácito de los actores
Formulación del problema del sistema de salud materna basado en evidencia
Desabasto de equipo y medicamento

- Falta de planeación en el ejercicio presupuestal y esquema de adquisiciones de bienes e insumos burocratizados y largos

Guerrero $\quad$ Falta de equipos y otros medios de comunicación entre unidades de salud y hospitales

- Falta de hospitales con capacidad resolutiva en emergencias obstétricas
Falta de hospitales con capacidad resolutiva ante la EO debido al desabasto de equipo y medicamento

- Asignación inequitativa y falta de planeación en el ejercicio del presupuesto estatal para la atención materna ${ }^{16}$

- Procedimientos administrativos deficientes y un esquema de adquisiciones burocratizado que ocasiona demoras e ineficiencia ${ }^{17}$

- Falta de cobertura de la atención de partos en las áreas rurales y escasa disponibilidad de recursos para cubrir guardias, lo cual se asocia con el incremento de las defunciones en fines de semana o periodos vacacionales $^{18}$

Capacitación, educación y promoción social

- Incorporación de personal con deficiencias técnicas

- Falta de disponibilidad y desconocimiento de normas, lineamientos

Estado de técnicos y manuales de procedimiento
México perio

- Notificación tardía de preclampsia o eclampsia en el primer nivel

Falta de resolución en las redes de EO

- Falta de hospitales con capacidad resolutiva en emergencias obstétricas

- Falta de equipos y otros medios de comunicación en unidades de salud y hospitales

- Falta de bancos de sangre y acceso limitado a hemoderivados, particularmente en zonas marginadas

- Fallas en la coordinación y seguimiento del sistema de referencia y contrarreferencia entre niveles de atención

Protocolos y guías clínicas

- Falta de unificación de criterios y organización ante la EO

- Notificación tardía de preeclampsia o eclampsia en el primer nivel

- Demasiado énfasis en el enfoque de riesgo obstétrico, omitiendo una visión integral de la salud materna
Insuficiente personal de salud capacitado y sensibilizado para brindar a las mujeres atención de calidad antes, durante y después del parto

- Incorporación al sistema de salud de personal con carencias técnicas ${ }^{19,20}$

- Falta de disponibilidad y desconocimiento de las normas, lineamientos técnicos y manuales de procedimiento, en particular durante la $\mathrm{EO}^{21,22}$

- Carencia de sistemas de incentivos para que el personal de salud asista a capacitaciones para mejorar la calidad de su atención ${ }^{23}$

Falta de resolución en las redes de EO

- Insuficiencia y desabasto en equipos, insumos y medicamentos, incluyendo el acceso a hemoderivados ${ }^{24}$

- Falta de cobertura del personal médico especializado las 24 horas, los 365 días del año ${ }^{25}$

- Deficiente calidad de la atención en el manejo de la EO y falta de capacidad resolutiva de las redes de salud materna o redes obstétricas ${ }^{26-28}$

- Redes rojas insuficientes o con un desempeño subóptimo*

- Falta de procesos de supervisión y eficacia productiva en la coordinación y comunicación en el primer nivel de atención ${ }^{29,30}$

Deficiencias en la calidad y la capacidad resolutiva de los servicios de salud que impiden la atención oportuna y eficiente de la EO

- La mayoría de las muertes maternas son evitables y suceden dentro del sistema de salud ${ }^{7,31}$

- Falta de conocimiento y la observancia de protocolos y guías clínicas como flujograma en vigencia ${ }^{21,22}$

- No adherencia a protocolos de atención y guías de manejo $0^{32}$

EO: Emergencia obstétrica

* Sesia P, García-Rojas M, García-Castellanos N, Carmona G, Sachse-Aguilera M. Resultados del monitoreo de las redes de servicios de salud materna del estado de Oaxaca. En: Freyermuth G, Sesia P, coords. Monitoreo, diagnósticos y evaluación en salud sexual y reproductiva: Nuevas experiencias de contraloría social. México: CIESAS/Comité Promotor por una Maternidad Segura. En prensa.

\section{Oaxaca}

La Cop priorizó la deficiencia de las redes de AEO. De acuerdo con la evidencia científica, uno de los problemas principales que enfrenta Oaxaca en salud materna tiene que ver precisamente con la baja capacidad resolutiva de los servicios de AEO en el segundo nivel de atención. Los hospitales enfrentan insuficiencia y desabasto en equipos, insumos y medicamentos, incluyendo el acceso a hemoderivados, ${ }^{24}$ sobre todo en el interior del estado. Se puntualizó y reafirmó la falta de cobertura del personal médico especializado las 24 horas los 365 días del año. ${ }^{25}$

En concordancia con el problema destacado por la Cop oaxaqueña sobre falta de equipos y otros medios de comunicación entre unidades de salud y hospitales, así como de coordinación y seguimiento entre niveles de atención, la literatura subraya la falta de capacidad 
resolutiva de las redes obstétricas como uno de los problemas más relevantes. ${ }^{26-28} \mathrm{El}$ monitoreo de las 18 "redes rojas" de salud materna en Oaxaca, realizado por el Comité Promotor por una Maternidad sin RiesgosOaxaca en 2009 y 2010, resaltó las deficiencias de esos servicios en el primer y segundo nivel de atención en cuanto a equipo, medicamentos, insumos y recursos humanos, lo que evidenció su incapacidad resolutiva ante las EO.*

Otro aspecto que se observó fue la falta de procesos de supervisión interna y externa que garanticen eficacia, especialmente en la articulación con el primer nivel de atención, ${ }^{29,30}$ por lo cual destaca la necesidad de seguimiento del sistema de referencia y contrarreferencia en EO, tal como lo manifiesta la Cop al elegir este problema.

\section{Veracruz}

El principal problema identificado por la Cop fue la existencia de deficiencias en la calidad y la capacidad resolutiva de los servicios de salud que impiden una AEO oportuna y eficiente. La revisión de la literatura permitió corroborar la importancia de este enfoque para evitar muertes maternas. ${ }^{19,31,32,42,43}$ En México la evidencia muestra que la mayoría de estos fallecimientos es evitable y sucede dentro del sistema de salud, ${ }^{7,25,31}$ lo cual enfatiza la falta de unificación de criterios y organización ante la EO. Al respecto, un tópico seleccionado por la Cop y que la bibliografía posicionó como central fue la no adherencia a protocolos de atención y guías de manejo. ${ }^{32}$ Estudios realizados en contextos similares al veracruzano refuerzan el argumento de que los profesionales de la salud no aplican con suficiente rigor las guías y manuales de procedimientos. ${ }^{21,22} \mathrm{La}$ Cop consideró como un problema el excesivo énfasis en el enfoque de riesgo para abatir la RMM, lo cual se fundamentó en los hallazgos sobre la imprevisibilidad de la EO aún cuando se lleve un embarazo con los controles adecuados. ${ }^{7,19,31,32,42,43}$

Llama la atención que al igual que en el Estado de México, en Veracruz tampoco es posible dar sustento científico al problema de la notificación tardía de la preclampsia y eclampsia.

\footnotetext{
* Sesia P, García-Rojas M, García-Castellanos N, Carmona G, SachseAguilera M. Resultados del monitoreo de las redes de servicios de salud materna del estado de Oaxaca. En: Freyermuth G, Sesia P, coords. Monitoreo, diagnósticos y evaluación en salud sexual y reproductiva: Nuevas experiencias de contraloría social. México: CIESAS/ Comité Promotor por una Maternidad Segura. En prensa.
}

\section{Discusión}

En la mayoría de los casos, la selección realizada por la Cop de cada estado fue corroborada con la evidencia científica. Esto pone de relieve la utilidad y relevancia de partir del conocimiento tácito de los actores con roles preponderantes y diferentes áreas de acción en el sistema de salud materna. ${ }^{10}$ La evidencia permitió profundizar en una primera formulación de los problemas, especificando, dando mayor enfoque y enriqueciendo su comprensión al poner de manifiesto importantes aspectos complementarios. La utilización de evidencia científica ofreció, además, elementos para dar el sostén necesario al seguimiento y la evaluación de las soluciones para los problemas escogidos.

En los cuatro estados las Cop priorizaron inicialmente problemas del sistema de salud relativos a la AEO. La literatura confirma la pertinencia de estos problemas, lo cual sugiere que esas comunidades están operando dentro de los ámbitos de las mejores recomendaciones internacionales. ${ }^{19}$ Por otra parte, en el ámbito nacional, el Convenio General de Colaboración para la AEO entre las diversas instituciones de salud pública ratifica dicho enfoque. ${ }^{6}$ El CNEGySR declara además, que "el gobierno mexicano ha optado por la AEO como la estrategia central para disminuir la mortalidad materna". ${ }^{7}$ Otra coincidencia destacable entre las cuatro Cop es su concordancia en centrarse en la provisión de servicios de salud para lograr el objetivo del milenio de acceso universal a la salud reproductiva, así como en la falta de resolución y deficiencias en la calidad de la atención prestada. Esta elección se sostiene en la evidencia que señala que el mayor número de muertes maternas se dan dentro de los sistemas de salud, con porcentajes que van desde 60 hasta $85 \%$, de acuerdo con las especificidades de cada estado. ${ }^{3}$

La Cop de Guerrero priorizó el problema de implementar y financiar un régimen de adquisiciones efectivo y sostenible. Este problema fue considerado el de mayor prioridad en el mapeo conceptual del conocimiento tácito de todos los actores estatales de manera conjunta. ${ }^{11}$ La literatura también sugiere la necesidad de abordar esta problemática de manera urgente para contar permanentemente con un cuadro básico de insumos indispensables para la $\mathrm{AEO}{ }^{34}$

La Cop mexiquense escogió un problema que no calificó como el más importante, pero sí entre los más factibles de resolver: la capacitación de los recursos humanos para atender la EO. La tendencia internacional pone énfasis en la necesidad de aumentar el porcentaje de partos atendidos por personal calificado como un factor relevante para disminuir la RMM. ${ }^{19}$ Por otra parte, entre las acciones más importantes para lograr 
esta disminución se describió mejorar las habilidades profesionales de quienes atienden las EO.$^{41}$ Cabe mencionar que la SSa está desarrollando un programa de capacitación en el Estado de México para el registro de estas emergencias. ${ }^{44}$

La Cop de Oaxaca abordó la EO de manera general pero focalizándose en las redes de atención. Para muchos autores es allí donde se cimenta la estrategia principal de AEO.* Por su parte, el CNEGySR ha elaborado numerosas recomendaciones basadas en evidencia y destaca su papel central en la disminución de la RMM. ${ }^{7}$ Cabe remarcar el enfoque que dio esta Cop sobre determinantes sociales a los cuales el sistema de salud suele no aportar soluciones efectivas. En concordancia con el enfoque de la EO, Oaxaca está reorganizando y compactando las redes de servicios para garantizar la disponibilidad de la AEO las 24 horas, los 365 días del año. ${ }^{\ddagger}$

En Veracruz, la Cop consideró prioritaria la necesidad de disponer de protocolos actualizados para una acción rápida y eficiente ante la $\mathrm{EO}$, y que sean conocidos y puestos en práctica por el personal de salud. Además, destaca la importancia de contar con un sistema de referencia y contrarreferencia efectivo y plausible. La literatura consultada por la Cop subraya la carencia de metodologías efectivas que garanticen la adherencia a los protocolos y guías de atención materna y perinatal, así como de decisiones administrativas y gerenciales que faciliten su cumplimiento. ${ }^{21,22}$

Destaca como única excepción que las Cop mexiquense y veracruzana no lograron dar un sustento claramente basado en la literatura sobre el problema de la notificación tardía de la preclampsia y eclampsia en el primer nivel. Cabe plantear dos explicaciones alternativas: que sea necesaria una búsqueda más exhaustiva de la literatura gris, más allá de la de acceso público en la red, incluyendo, por ejemplo, las recomendaciones de los comités estatales de mortalidad materna; o bien, es posible que no exista documentación suficiente para sustentar la validez de este problema, lo cual manifesta la necesidad de investigar más al respecto.

El proyecto deja ver la factibilidad de analizar las formulaciones de los problemas de salud desde la perspectiva de la traducción del conocimiento con la finalidad de acercar recomendaciones oportunas y pertinentes a los decisores, pero también adecuadas a

\footnotetext{
* Sesia P, García-Rojas M, García-Castellanos N, Carmona G, SachseAguilera M. Resultados del monitoreo de las redes de servicios de salud materna del estado de Oaxaca. En: Freyermuth G, Sesia P, coords. Monitoreo, diagnósticos y evaluación en salud sexual y reproductiva: Nuevas experiencias de contraloría social. México: CIESAS/ Comité Promotor por una Maternidad Segura. En prensa.

₹ Servicios de Salud de Oaxaca. Plan estratégico para la operación de redes de servicios en el estado de Oaxaca, 2012. Presentación PowerPoint, 13 de enero.
}

las realidades y diferentes necesidades. La identificación de las Cop de la mejor evidencia en el ámbito estatal fortalece su capacidad para identificar soluciones apropiadas y les permitirá relacionarse con las autoridades federales con mejores bases de conocimiento. Una de las mayores fortalezas en el desarrollo del proyecto fue la recopilación de la bibliografía de diversa índole y el análisis de su pertinencia ante las realidades estatales. De este modo se hallaron incluso referencias sobre países con contextos aparentemente diferentes a México, pero adecuados a situaciones específicas de los estados, de acuerdo con la mirada de las Cop. Esto permite suponer que la metodología puede ser aplicable a diferentes países sobre la base del conocimiento de primera mano de las comunidades familiarizadas tanto con la problemática escogida como con las situaciones contextuales particulares.

La investigación tuvo como limitante no haber incluido a representantes de la sociedad civil ni a las autoridades municipales y de otros sectores de gobierno, debido a las restricciones propias de la organización de las Cop. Los problemas percibidos, así como su reformulación, tienden a enfocarse en los determinantes más accesibles del sistema de salud. No obstante, el caso de Oaxaca demuestra que la participación de investigadores dentro de la Cop permite ampliar la perspectiva. Posteriormente será importante dar cuenta de éstas y otras fortalezas y debilidades del proyecto para enriquecer futuras experiencias similares.

Este esfuerzo de gestión del conocimiento realizado por Cop estatales destaca la importancia de complementar el conocimiento tácito con la evidencia científica para enfocar la formulación de los problemas del sistema de salud hacia soluciones efectivas adecuadas a cada contexto.

\section{Agradecimientos}

Los autores agradecen la participación de las Cop de los estados en la búsqueda y selección de artículos que conforman la evidencia científica presentada. Se agradece asimismo el financiamiento de la Alianza para la Investigación en Políticas y Sistemas de Salud de la OMS.

Declaración de conflicto de intereses. Los autores declararon no tener conflicto de intereses.

\section{Referencias}

I. Organización de las Naciones Unidas. Objetivos de Desarrollo del Milenio. 2000. [Consultado 2010 junio II]. Disponible en: http://www. un.org/spanish/milenio/ares552.pdf 
2. Dirección General de Epidemiología, Subsecretaría de Prevención y Promoción de la Salud, Secretaría de Salud. Informe semanal de vigilancia epidemiológica. [Actualizado 20II diciembre 28; consultado 2012 jun 12]. Disponible en: http://www.omm.org.mx/images/stories/MMaternas_sem52.pdf

3. Freyermuth G, Luna M. Mortalidad materna en México. Numeralia 2010. México: Observatorio de Mortalidad Materna, 201 I.

4. Centro Nacional de Equidad de Género y Salud Reproductiva, Secretaría de Salud. Programa de acción Arranque Parejo en la Vida. México: SSa, 2003.

5. Seguro Popular de Salud. Embarazo Saludable. México: Secretaría de Salud, 2009. [Consultado 201I julio I8]. Disponible en: http://www. seguro-popular.salud.gob.mx/index.php?option=com_content\&view=articl e\&id $=25$ :embarazosaludable $\&$ catid $=3$ :embarazosaludable\&ltemid $=190$ 6. Secretaría de Salud. Convenio general de colaboración para la atención de la emergencia obstétrica 2009. [Consultado $201 \mathrm{I}$ junio 28]. Disponible en: http://www.dgplades.salud.gob.mx/descargas/convenios_emergencias_obs/ceo_conv.pdf

7. Centro Nacional de Equidad de Género y Salud Reproductiva, Secretaría de Salud. Estrategia integral para reducir la mortalidad materna en México. 2009. [Consultado 20II junio 17]. Disponible en: http://www. coneval.gob.mx/cmsconeval/rw/resource/coneval/info_public/Estrategia\%20Integral.pdf?view=true

8. Comité Promotor por una Maternidad Segura. Cuarta Reunión Técnica de Promoción en Salud Materna; 20II. [Consultado 20II agosto 23] Disponible en: http://maternidadsinriesgos.org.mx/web/index.php/cuartareunion-tecnica

9. Becerril-Montekio V, Gonzalez-Block MA, Rouvier M. Atención problemas del sistema de salud maternal. Resultados del proyecto: Investigación para el Desarrollo de los Sistemas Estatales de Salud. INDESES II. Resumen ejecutivo. México: Instituto Nacional de Salud Pública, 20I I 10. Gonzalez-Block MA, Rouvier M, Becerril V, Sesia P. Mapping of health system functions to strengthen priority programs. The case of maternal health in Mexico. BMC Public Health [serie en internet] 20I I. [Consultado 201 I junio 19]. Disponible en: http://www.biomedcentral.com/l47I2458/II/164

I I. Rouvier M, Gonzalez-Block MA, Becerril-Montekio V, Sesia P, Duarte MB, Flores-Collins E. Mapeo de problemas para la atención a la salud materna por actores estatales y federales. Salud Publica Mex 20I I;53:48-56. 12. Lavis JN. A brief guide to problem definition. Hamilton: Program in Policy Decision-Making, McMaster University's Program in PolicyDecision-Making, 2006.

13. Lavis JN, Wilson M, Oxman AD, Lewin S, Fretheim A. Support tools for evidence-informed health policymaking. 4. Using research evidence to clarify a problem. Health Res Policy Syst 2009;7 suppl I:S4 DOI: I0.1 I86/1478-4505-7-SI-S4

14. Wenger E, McDermott R, Snyder WM. Cultivating communities of practice. Boston: Harvard Business School Press, 2003.

15. Glaser B, Strauss A. The discovery of grounded theory. Strategies for qualitative research. New York: Aldine de Gruyter, 1967.

16. Díaz D, coord. Muerte materna y presupuesto público en los estados de Chiapas, Guerrero y Oaxaca. México: Fundar, 2004.

17. Subdirección de Innovación y Calidad, Secretaría de Salud. Programa de acción específico 2007-2012. Mejora del acceso a medicamentos México: Secretaría de Salud. [Consultado 2010 octubre I4]. Disponible en: http:// www.dgplades.salud.gob.mx/descargas/biblio/PAE_Med_actualizado.pdf 18. Subsecretaría de Prevención y Promoción de la Salud, Secretaría de Salud. Programa de acción específico 2007-2012. Arranque Parejo en la Vida. [Consultado 2010 junio II]. Disponible en: http://www.generoysaludreproductiva.salud.gob.mx/descargables/programas/paes/pae_apv.pdf 19. United Nation Population Found. Ninguna mujer debería morir al dar a luz. United Nation Population Found, 2008. [Consultado 2010 noviembre 30]. Disponible en: http://www.unfpa.org/safemotherhood
20. Steven A, Wong-Blandón YC, McCaw-Binns A, Sandino I, Urbina L, Rodriguez $C$ et al. Are skilled birth attendants really skilled? A measurement method, some disturbing results and a potential way forward. B World Health Organ 2007;85(I0):783-790.

21. Munera $\mathrm{H}$, Piedrahita M. Evaluación de la baja adherencia a guías médicas implementadas en la ESE Hospital San Vicente de Paúl de Caldas Antioquia primer semestre. Medellín: Universidad de Antioquia, Facultad Nacional de Salud Pública "Héctor Abad Gómez", 2008. [Consultado 2010 noviembre 22]. Disponible en: http://tesis.udea.edu.co/dspace/ bitstream// 0495/43I///EvaluacionAdherenciaGuiasMedicas.pdf 22. Rojas-Higuera R, Londoño-Cardona JG, Arango-Gómez F. Prácticas clínicas en el cuidado de gestantes y recién nacidos en algunos hospitales de Bogotá, Colombia. Rev Salud Pública 2006;8(3):223-234.

23. Mayca J, Palacios-Flores E, Medina A, Velásquez J, Castañeda D. Percepciones del personal de salud y la comunidad sobre la adecuación cultural de los servicios materno-perinatales en zonas rurales andinas y amazónicas de la región Huánuco. Rev Perú Med Exp Salud Pública 2009;26(2):145-160.

24. Dogba M, Fournier P. Human resources and the quality of emergency obstetric care in developing countries: A systematic review of the literature. Hum Resources Health 2009;7:7.

25. Freyermuth $\mathrm{G}$. Mortalidad materna. Inequidad institucional y desigualdad entre mujeres. México: Coneval, 20I0:23. [Consultado 2010 julio II]. Disponible en: http://www.coneval.gob.mx/contenido/info_public/68I5.pdf 26. Bulatao RA, Ross JA. Rating maternal and neonatal health services in developing countries. B World Health Organ 2002;80:721-727.

27. De Brouwere V, Tonglet R, Van Lerberghe W. Strategies for reducing maternal mortality in developing countries: What can we learn from the history of the industrialized West? Trop Med Int Healt 1998;3:77I-782. 28. Ronsmans C, Graham WJ. Maternal Survival I. Maternal mortality: Who, when, where and why. Lancet 2006;368: I 189-1200.

29. Campbell OM, Graham W]. Maternal Survival 2. Strategies for reducing maternal mortality: Getting on with what works. Lancet 2006;368: I284- I 299 .

30. Paxton A, Maine D, Freedman L, Fry L, Lobis S. The evidence for emergency obstetric care. Int J Gynecol Obstet 2005;88: I8I-193. 31. Núñez-Urquiza RM, Duarte-Gómez MB, Javier-Marin L, ArroyoRomán L. Más allá de las cifras detección de eslabones críticos en los procesos de atención para disminuir muertes maternas. Género y salud en cifras. México: Secretaría de Salud, 2005:5-I5.

32. Anwar I, Kalim N, Koblinsky M. Quality of obstetric care in publicsector facilities and constraints to implementing emergency obstetric care services: Evidence from high- and low-performing districts of Bangladesh. J Health Popul Nutr 2009;2:I39-I55.

33. Borghi J, Sabina M, Ronsmans C, Killewo J. Comparison of costs of home and facility-based basic obstetric care in rural Bangladesh. J Health PopulNutr 2010;28(3):286-293.

34. Johns B, Kristjana S, Fogstad H, Zupan J, Mathai M, Tan-Torres Edejer $\mathrm{T}$. Estimated global resources needed to attain universal coverage of maternal and newborn health services. B World Health Organ 2007;85(4):256-263.

35. Padmanaban P, Parvathy Sankara R, Mavalankar DV. Innovations and challenges in reducing maternal mortality in Tamil Nadu, India. J Health Popul Nutr 2009;27(2):202-219.

36. Borghi J, Ensor T, Somanathan A, Lissner C, Mills A. Maternal Survival 4. Mobilising financial resources for maternal health. Lancet 2006;368: | 457-| 465

37. Centro de Estudios de Estado y Sociedad. Mortalidad materna en la Argentina. Diagnóstico para la reorientación de políticas y programas en salud [Resumen ejecutivo 2010. [Consultado 2010 noviembre 5]. Disponible en: http://www.cedes.org/informacion/index2.html

38. Kowalewski M, Jahn A. Health professionals for maternity services: Experiences on covering the population with quality maternity care. En: Van Lerberghe W, Kegels G, De Brouwere V, eds. Safe motherhood 
strategies: A review of the evidence. Studies in Health Services Organization \& Policy, 2001.

39. Padilla K. La muerte materna en Nicaragua. La tragedia ignorada de la mortalidad materna nos hace una pregunta fundamental. ¿En cuánto valoramos la vida de una mujer? Managua: IPAS, 2003.

40. United Nations Development Programme. MDG 5: Improve maternal health. En: Malaysia. Achieving the Millennium Development Goals.

Successes and challenges. Kuala Lumpur: United Nations Country Team Malaysia, 2005. [Consultado 2010 julio 20]. Disponible en: http://www. undp.org.my/uploads/mdg5.pdf

4I. Cárdenas R. Acciones y programas para la reducción de la mortalidad materna: ¿Qué necesitamos hacer? Congreso de Investigación en Salud Pública. Salud Publica Mex 2007;edición especial I:231-233.
42. Engender Health, AMDD Mailman School of Public Health of the Columbia University, United States Agency for International Development, eds. Mejoramiento de la calidad para atención obstétrica de emergencia. New York: 2005. [Consultado 2010 noviembre II]. Disponible en: http:// www.engenderhealth.org/files/pubs/maternal-health/QI-for-EMOCHerramientas.pdf

43. lyengar K, lyengar SD, Suhalka V, Dashora K. Pregnancy-related deaths in rural Rajasthan, India: Exploring causes, context, and care-seeking through verbal autopsy. J Health Popul Nutr 2009;27(2):293-302. 44. Dirección General de Planeación y Desarrollo en Salud, Secretaría de Salud. 20II. [consultado 2012 jun I2]. Disponible en: http://www. dgplades.salud.gob.mx/interior/presentaciones.html 